\title{
High-resolution coherent backscatter interferometric radar images of equatorial spread F using Capon's method
}

\author{
Fabiano S. Rodrigues ${ }^{1}$, Eurico R. de Paula ${ }^{2}$, and Gebreab K. Zewdie ${ }^{1}$ \\ ${ }^{1}$ W. B. Hanson Center for Space Sciences, The University of Texas at Dallas, Richardson, TX, USA \\ ${ }^{2}$ Instituto Nacional de Pesquisas Espaciais - INPE, São José dos Campos, SP, Brazil \\ Correspondence to: Fabiano S. Rodrigues (fabiano@utdallas.edu)
}

Received: 9 August 2016 - Revised: 20 February 2017 - Accepted: 23 February 2017 - Published: 14 March 2017

\begin{abstract}
We present results of Capon's method for estimation of in-beam images of ionospheric scattering structures observed by a small, low-power coherent backscatter interferometer. The radar interferometer operated in the equatorial site of São Luís, Brazil $\left(2.59^{\circ} \mathrm{S}, 44.21^{\circ} \mathrm{W},-2.35^{\circ}\right.$ dip latitude). We show numerical simulations that evaluate the performance of the Capon method for typical $F$ region measurement conditions. Numerical simulations show that, despite the short baselines of the São Luís radar, the Capon technique is capable of distinguishing localized features with kilometric scale sizes (in the zonal direction) at $\mathrm{F}$ region heights. Following the simulations, we applied the Capon algorithm to actual measurements made by the São Luís interferometer during a typical equatorial spread F (ESF) event. As indicated by the simulations, the Capon method produced images that were better resolved than those produced by the Fourier method. The Capon images show narrow (a few kilometers wide) scattering channels associated with ESF plumes and scattering regions spaced by only a few tens of kilometers in the zonal direction. The images are also capable of resolving bifurcations and the $\mathrm{C}$ shape of scattering structures.
\end{abstract}

Keywords. Radio science (interferometry; ionospheric physics; instruments and techniques)

\section{Introduction}

Equatorial spread $\mathrm{F}$ (ESF) refers to a broad spectrum of electron density irregularities occurring in the equatorial and low-latitude $\mathrm{F}$ region ionosphere. ESF is associated with interchange plasma instabilities, which can create large-scale (tens of kilometers) sizes plasma density perturbations. Secondary plasma instabilities then create smaller-scale (down to centimeter) irregularities (Woodman, 2009). While the overall features of ESF are well-understood, we still seek a better understanding of the processes leading to the variability in the generation, development and decay of ESF events. In addition to the scientific aspect of studying instabilities in space plasmas, the investigation of ESF is also motivated by its impact on the propagation of trans-ionospheric radio waves such as those used for remote sensing, navigation and communication (e.g., Kintner et al., 2007).

Ground-based ionospheric radars have contributed significantly to our understanding of ionospheric plasma and irregularities. Ionospheric radars are used to measure the scatter from electron density irregularities matching the Bragg scatter condition. Because these radars usually operate in the VHF and UHF bands, the scatter comes from irregularities in scale sizes ranging from a few meters down to a few tens of centimeters.

Incoherent scatter radars are high-power, large-aperture systems that can measure extremely weak echoes produced by thermal electron density fluctuations. Coherent scatter radars, on the other hand, are smaller systems capable of measuring echoes produced by non-thermal electron density fluctuations, that is, fluctuations caused by plasma instabilities. Conventional coherent radar measurements are capable of providing information about the altitudinal distribution of irregularities, as a function of time, as well as the mean radial phase velocity of the irregularities producing the echoes. Additionally, conventional coherent radars provide information about the strength of plasma turbulence at the wavelength matching the Bragg condition.

Radar measurements made using spaced antennas can provide additional information about the irregularities causing echoes (e.g., Kudeki and Franke, 1986). Using multiple 
baselines, one can estimate the two-dimensional distribution (zonal distance versus height) of the scatterers responsible for the observed echoes using interferometric imaging techniques. Coherent scatter radar (in-beam) images can help us understand the underlying plasma processes responsible for ionospheric disturbances, including those associated with ESF. Radar images can also be used to help validate theoretical predictions of equatorial spread F (e.g., Hysell et al., 2014).

Coherent backscatter radar imaging has been used for investigations of equatorial spread $\mathrm{F}$. These investigations have been performed, predominantly, in the Peruvian longitude sector using measurements made by the radar and antenna modules of the Jicamarca Radio Observatory (e.g., Kudeki and Sürücü, 1991; Hysell and Woodman, 1997; Hysell and Chau, 2006; Harding and Milla, 2013) with only a couple of exceptions (Rodrigues et al., 2008, 2012). Initial studies used Fourier-based analyses of the measurements (Kudeki and Sürücü, 1991). More advanced techniques, using the maximum entropy algorithm (MaxEnt), were implemented by Hysell (1996). Since then, the MaxEnt technique has been improved, well tested and is now commonly used at Jicamarca. Palmer et al. (1998) and Yu et al. (2000) have also proposed that the Capon method can be used to create interferometric images of scattering structures producing coherent scatter echoes. The implementation of the method is simple, not computationally intensive and produces images of the scattering regions with a much higher spatial resolution than those produced by the Fourier method. Despite the encouraging results of Palmer et al. (1998) and Yu et al. (2000), limited efforts have been made to use the Capon method in ionospheric studies. An exception is the work of Chau and Woodman (2001), which used the Capon method and other techniques to create radar images of electrojet irregularities.

In the present study, we revisit the Capon method and investigate its performance when applied to measurements made by a small, low-power coherent backscatter radar interferometer located at the equatorial site of São Luís, Brazil. We show that the method can produce high-resolution images, which reproduce features predicted by numerical simulations and previous observations of interchange plasma instabilities. This report is presented as follows: Sect. 2 describes the setup used by the São Luís radar for interferometric measurements. Section 3 provides information about the Fourier and Capon methods for interferometric imaging. In Sect. 4, we present and discuss the results of analyses. Numerical simulations are used to evaluate the performance of the Capon method. We also present and discuss the results of applying the Capon algorithm to actual measurements made by the São Luís radar during a typical pre-midnight ESF event. The features in the scattering structures resolved by the Capon method are discussed in light of our current understanding of ESF. Finally, Sect. 5 summarizes the main results of our study.

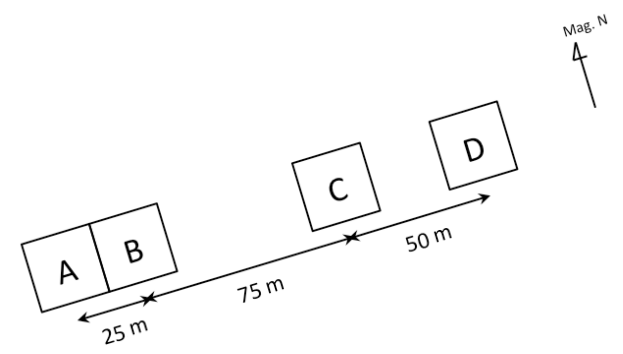

Figure 1. Diagram describing the distribution of the antenna sets used for the interferometric observations. Each antenna set is formed by a four by four array of Yagi antennas. A, B, C and D represent the antenna sets. Transmissions are made with sets $\mathrm{A}$ and B. Reception is made with all of the antenna sets. The magnetic declination is approximately $21^{\circ} \mathrm{W}$.

\section{Measurements}

The measurements available for this study were made by a $30 \mathrm{MHz}$ coherent backscatter radar interferometer. The radar was installed at the equatorial site of São Luís, Brazil $\left(2.59^{\circ} \mathrm{S}, 44.21^{\circ} \mathrm{W},-2.35^{\circ}\right.$ dip latitude) and operated between 2000 and 2012. The São Luís radar is a low-power radar system that was used for studies of equatorial ionospheric irregularities in the $\mathrm{E}$ and $\mathrm{F}$ regions (de Paula and Hysell, 2004; Rodrigues et al., 2008; Shume et al., 2014). Starting in 2005, the radar was equipped with four independent antenna sets connected to four receivers. Each antenna set is formed by an array of four by four Yagi antennas. The arrays have been placed non-uniformly in the magnetic zonal direction for radar imaging studies of scattering layers. Figure 1 shows the distribution of the antennas in the magnetic zonal direction. The longest baseline is $150 \mathrm{~m}$, that is $15 \lambda$, where the $\lambda$ is the wavelength of the $30 \mathrm{MHz}$ radar signal. Two $4 \mathrm{~kW}$ transmitters are available for observations and were normally used for $\mathrm{F}$ region measurements.

For $\mathrm{F}$ region observations, we used 28 bit coded pulses, with a $9.33 \mathrm{~ms}$ inter-pulse period (IPP). The baud length and sampling were $2.5 \mathrm{~km}$. A total of 250 samples were collected per IPP. This observation setup allowed us to make measurements of the $\mathrm{F}$ region from 200 to $825 \mathrm{~km}$ altitude with a range resolution of $2.5 \mathrm{~km}$.

\section{Analysis}

Coherent scatter radar imaging techniques have been used to determine the distribution of scatterers as a function of height and zenith angle $(\psi)$ (e.g., Kudeki and Sürücü, 1991; Woodman, 1997; Palmer et al., 1998; Hysell and Chau, 2006). The real-valued, continuous function representing this distribution is referred to as Brightness distribution $B(\psi)$, and it is computed for each range gate.

The brightness distribution is closely related to the cross correlation of scattered signals received by antennas spaced 
by a distance $d$. A commonly used estimator for the normalized cross correlation of signals measured by spaced antennas 1 and 2 is given by

$V(k d)=\frac{\left\langle v_{1} v_{2}^{*}\right\rangle}{\sqrt{\left(\left\langle\left|v_{1}\right|^{2}\right\rangle-N_{1}\right)\left(\left\langle\left|v_{2}\right|^{2}\right\rangle-N_{2}\right)}}$,

where the angle brackets represent an ensemble average. In practice, time averages are used, with $v_{1}$ and $v_{2}$ being complex-valued voltage samples measured by antennas 1 and 2 , respectively. $N_{1}$ and $N_{2}$ are estimates of the noise power in each receiver, and $k=\frac{2 \pi}{\lambda}$ with $\lambda$ is the wavelength of the radar signal.

In imaging, the spatial cross-correlations $V(k d)$ are referred to as visibility measurements. For the case of ionospheric imaging, where the scatters are assumed to be twodimensional due to the nature of field-aligned irregularities, the relationship between visibility and brightness is given by

$V(k d) \approx \int_{-\infty}^{+\infty} B(\psi) e^{j k d \psi} d \psi$

The visibility is the correlation of the scattered signals received by antennas spaced by a distance $d$.

One can recognize that Eq. (2) represents a Fourier-type integral, and, therefore, the brightness can be estimated from the visibility function using the inverse Fourier transform:

$B(\psi)=\int_{-\infty}^{+\infty} V(k d) e^{-j k d \psi} d(k d)$.

In the practical case of a finite number of receivers $(n)$, the Fourier transform estimate of the brightness distribution $\left(\hat{B}_{\mathrm{f}}\right)$ can be obtained from (Yu et al., 2000)

$\hat{B}_{\mathrm{f}}(\psi)=\boldsymbol{w}_{\mathrm{f}}^{\mathrm{H}} \mathbf{V} \boldsymbol{w}_{\mathrm{f}}$,

where $\mathrm{H}$ denotes the Hermitian operator, $\mathbf{V}$ is the $n \times n$ visibility matrix, and $\boldsymbol{w}_{\mathrm{f}}$ is the weight (or pointing) vector given by

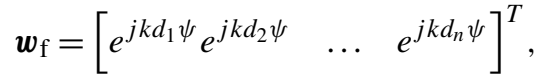

where $d_{i}$ is the distance of receiver $i$ to a reference point and $T$ denotes the transpose operator.

Ionospheric radar images are obtained by computing the brightness function for each range gate independently. The images, therefore, represent the distribution of ionospheric irregularities causing echoes, as a function of range and zenith angle. The brightness functions can also be obtained for different Doppler shifts adding a new dimension to the images. A sequence of images can be used to track the appearance, development and decay of scattering regions and to understand the dynamics of the irregularity structures.

\section{The Capon method}

The angular resolution of the images obtained with the Fourier method is limited by the length of the longest baseline. Palmer et al. (1998) proposed a new technique to obtain images with higher angular resolution than those provided by the Fourier method. The technique is based on the spectral analysis approach proposed by Capon (1969). The method selects the set of weights $\left(\boldsymbol{w}_{\mathrm{c}}\right)$ that will not only provide a high-resolution spectrum but will also suppress the effects of interfering signals. The set of weights is obtained by setting the search for the brightness distribution as a constrained optimization problem. The Capon weights are such that they minimize interference from signals outside the direction of interest. A detailed description of the derivation of the Capon method is given by Palmer et al. (1998) and Yu et al. (2000). They show that the Capon set of weights is given by

$\boldsymbol{w}_{\mathrm{c}}=\frac{\mathbf{V}^{-1} \boldsymbol{e}}{\boldsymbol{e}^{\mathrm{H}} \mathbf{V}^{-1} \boldsymbol{e}}$,

where $\boldsymbol{e}=\left[\begin{array}{lll}e^{j k d_{1} \psi} e^{j k d_{2} \psi} & \ldots & e^{j k d_{n} \psi}\end{array}\right]^{\mathrm{T}}$. Note that, unlike the conventional Fourier transform, the Capon weights depend on the visibility data. Therefore, the Capon technique can be described as an adaptive method. The Capon weights lead to an estimate of the brightness function given by

$\hat{B}_{\mathrm{c}}(\psi)=\frac{1}{\boldsymbol{e}^{\mathrm{H}} \mathbf{V}^{-1} \boldsymbol{e}}$.

Despite being a technique that is computationally simple to implement and to execute and despite the encouraging results obtained by Palmer et al. (1998) and Yu et al. (2000), the Capon method has not yet been widely used in ionospheric irregularity studies. The potential of the technique and availability of measurements motivated our investigation of the performance of the Capon method when applied to measurements made by a low-power, short-baseline radar interferometer such as those provided by the São Luís radar.

\section{Results and discussion}

\subsection{Numerical simulations}

Before obtaining imaging results from actual measurements, we investigated the ability of the Capon method to produce accurate estimates of the distribution of the irregularities. We considered the specific case of the São Luís baseline setup and created synthetic visibility distributions for different scattering geometries (brightness distributions) under different levels of measurement errors.

Measurement errors for normalized correlations were taken into account considering typical SNR values observed during ESF events and the number of incoherent integrations $(K)$ used in the ESF observations made by the São Luís radar. 

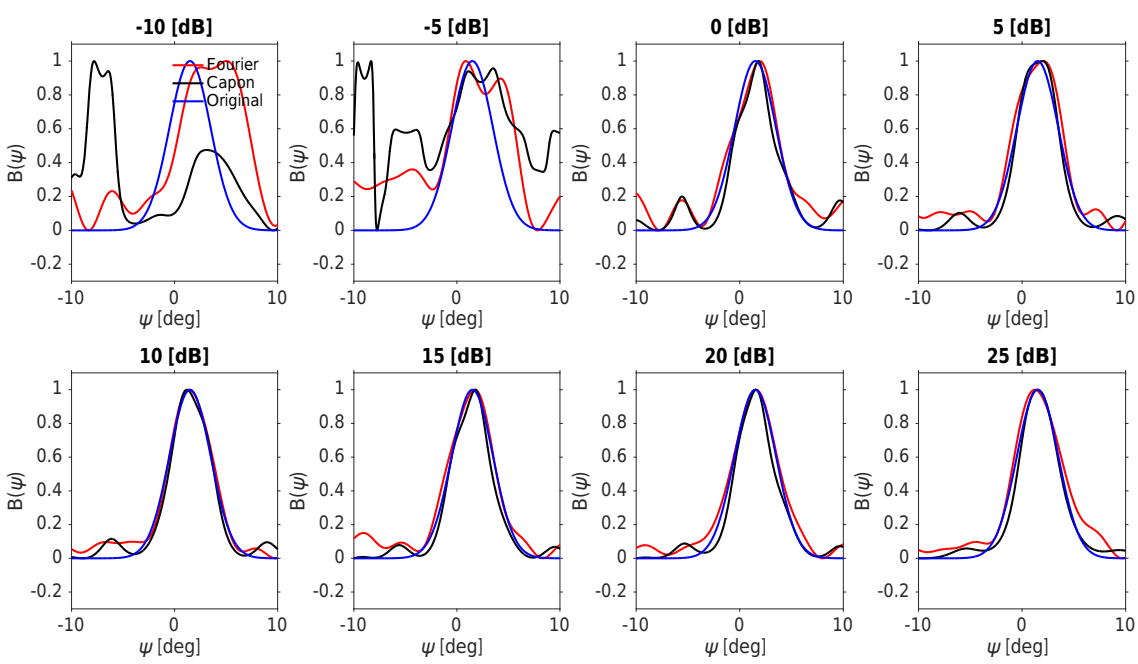

Figure 2. Numerical simulations of the Fourier and Capon methods under different SNR conditions. The simulated brightness function is described by a Gaussian function centered at $1.5^{\circ}$ with an angular width of $2^{\circ}$.

The expected value for the error variances of the normalized correlation functions $(\rho)$ are given by (e.g., Farley, 1969; Farley and Hysell, 1996)

$$
\begin{aligned}
\left\langle|\hat{\rho}-\langle\hat{\rho}\rangle|^{2}\right\rangle & \approx \frac{1}{K}\left(\frac{S+N}{S}\right)^{2}\left[1+\frac{1}{2}|\hat{\rho}|^{2}\left(1+\left|\frac{S}{S+N} \rho\right|^{2}\right)\right. \\
& \left.-2 \frac{S}{S+N}|\rho|^{2}\right]
\end{aligned}
$$

where $K=800$, for typical $\mathrm{F}$ region observations made with the São Luís interferometer. For this analysis, we assumed that the errors in noise estimation were negligible and that the errors in the visibility estimates were statistically independent.

In order to evaluate the potential of the Capon method, two scenarios were considered in our simulation analysis. These two scenarios illustrate the weaknesses and strengths of the Capon method.

The first simulation scenario considered a broad distribution of scatterers (brightness) that is described by a Gaussian function, centered at $1.5^{\circ}$ zenith angle. The Gaussian function has an angular width $(\sigma)$ of $2^{\circ}$. Figure 2 shows the results of the inversions of this simulated brightness function using the Capon method for different SNR conditions. It also shows results of the inversion using the Fourier method and the true brightness function. Each panel only shows the result of a single realization of the inversion for the SNR condition indicated at the top of each panel. The results shown in Fig. 2 indicate that, for the São Luís setup and a broad brightness distribution function, the Fourier and Capon methods have similar performances for most of the SNR values considered in the simulations. Both methods are capable of retrieving adequate information about the brightness distribution, at least for conditions of $\mathrm{SNR}>-5 \mathrm{~dB}$. For $\mathrm{SNR} \leq-5 \mathrm{~dB}$, little similarity (low correlation) was found between the inversions and the true brightness distribution.

The second simulation scenario presented here considered a scattering region that is described by two Gaussian distributions centered at $\pm 1.5^{\circ}$ off zenith. In this case, the ability of the Capon method to resolve closely spaced, narrow features was investigated, and the angular width of each scattering center was set to $0.2^{\circ}$. Figure 3 shows the results of the simulations for the narrow brightness distributions. In this simulation scenario, the Capon technique shows its strength, that is, the high resolution. The Capon method was able to better resolve the narrow structures than the Fourier method even under conditions of very low SNR $(\sim-5 \mathrm{~dB})$. The length of

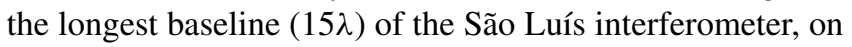
the other hand, severely limits the angular resolution of the Fourier inversions, and the narrow spectral features cannot be properly resolved even under high SNR conditions.

Figure 4 summarizes our simulation results and our evaluation of the performance of the Capon and Fourier methods as a function of SNR. The performance of the inversion is quantified by the normalized correlation (Pearson correlation coefficient) of the inverted brightness and the true brightness. It shows the average value of the normalized correlation of inversions with the true brightness distribution as a function of SNR conditions. The average for each SNR is the result of 1000 realizations. The results are shown for the two scattering distribution scenarios described above. The top panel shows the results for one single broad brightness distribution and the bottom panel shows the results for two narrow brightness centers.

Figure 4a shows that, for a broad scattering distribution and the setup of the São Luís interferometer, the Capon method does not outperform the Fourier method. In fact, its ability to recover the correct brightness distribution is 

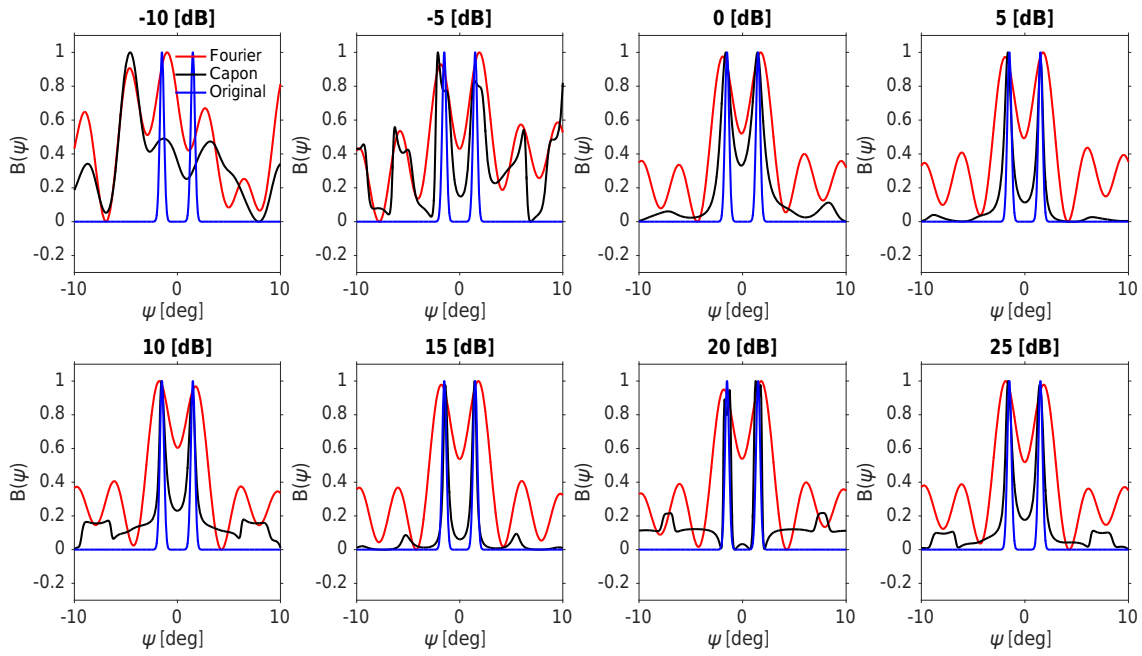

Figure 3. Numerical simulations of the Fourier and Capon methods under different SNR conditions. The simulated brightness function is described by two Gaussian functions centered at $\pm 1.5^{\circ}$ and an angular width of $0.2^{\circ}$.

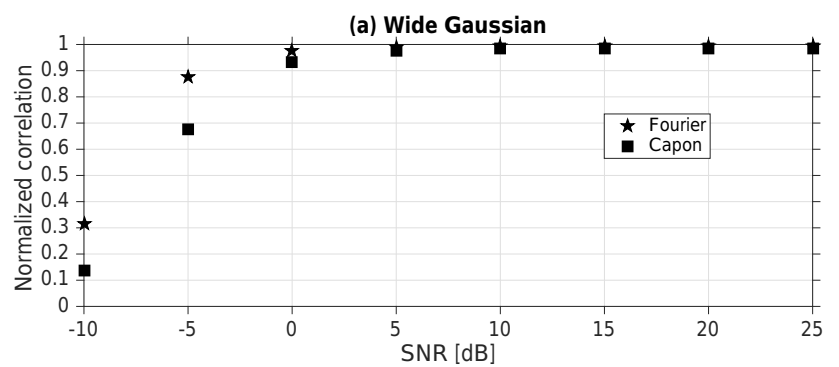

(b) Narrow Gaussians

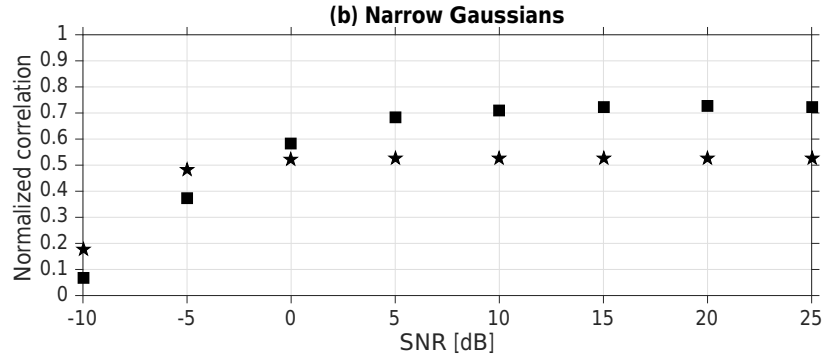

Figure 4. Simulation results describing the performance of the Fourier and Capon methods as a function of SNR conditions for two scattering distribution scenarios: (a) the brightness distribution is described by a single wide Gaussian function and (b) by two narrow Gaussian functions. Each correlation estimate is an average of correlations obtained for 1000 simulations.

slightly inferior for most SNR conditions. At very low SNR conditions ( $\mathrm{SNR}<0 \mathrm{~dB}$ ), the ability of the Capon method to recover the brightness distribution decreases substantially, and is more clearly outperformed by the Fourier method. Both methods, nevertheless, are capable of recovering the correct brightness with high accuracy given echoes with adequate SNR. The normalized correlations are greater than 0.9 for $S N R \geq 0 \mathrm{~dB}$.
Figure $4 \mathrm{~b}$ shows simulation results for the case of two narrow scattering structures. The results show that, for this case, the Capon method provides more accurate inversions than the Fourier method for SNR levels greater than $-5 \mathrm{~dB}$. The accuracy of the inversion does not exceed a correlation of approximately 0.72 even for SNR $=20 \mathrm{~dB}$. The accuracy, however, could be improved further by reducing the error in the correlation estimates. The error can be reduced, for instance, by adjusting the IPP and increasing the number of incoherent integrations $(K)$.

The results shown in Fig. 4 confirm the inferences we made based on simulations presented in Figs. 2 and 3. The Capon method shows its strength when estimating the brightness distribution of localized scatterers. It is capable of identifying multiple structures with widths that are just a fraction of a degree and that cannot be adequately retrieved with the Fourier method. The ability of the Capon method to produce high-resolution radar images agrees with results of previous studies (e.g., Yu et al., 2000; Chau and Woodman, 2001). Yu et al. (2000), in particular, found that even an interferometer with a limited number of short baselines can produce estimates of the brightness distribution that are highly correlated with the true distribution. Similar to our results, they also found that, for localized scatterers, the Capon method outperforms the Fourier method especially when conditions of SNR $>0 \mathrm{~dB}$ are considered. Yu et al. (2000) showed that, even for conditions of high SNR and a large number of wellspaced receivers, the correlations between Capon and true brightness distributions can be significantly less than 1 . We also found that even for SNR $=20 \mathrm{~dB}$ the normalized correlation is around 0.72. Finally, Yu et al. (2000) also found that the performance of the Fourier method does not change much for SNR $>-5 \mathrm{~dB}$. 

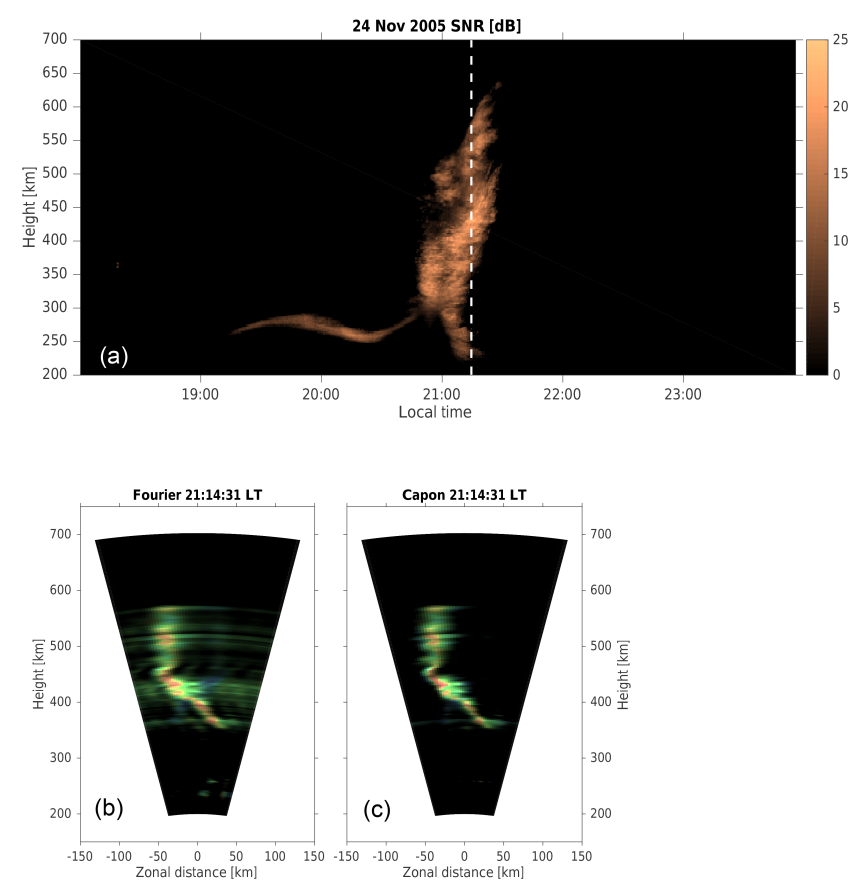

Figure 5. Panel (a) shows the range-time-intensity map of F region observations made by the São Luís radar on 24 November 2005. Panels (b, c) show the images obtained with the Fourier transform (b) and Capon methods (c) for observations from 21:14:31 LT. Zonal distance is positive to the east. The estimated images are scaled by the SNR of the echoes. The Doppler information is encoded in the pixel colors. The green components represent small mean Doppler velocities. Red and blue components represent irregularities moving away and towards the radar, respectively.

\subsection{In-beam radar images}

We have also analyzed the results of the Capon method applied to actual interferometric measurements made by the São Luís radar. We applied our Fourier and Capon algorithms to ESF measurements made by the São Luís radar. For this study, we present results of our analysis of a well-developed ESF event observed by the São Luís radar on the night of 24 November 2005. We present and discuss some of the features of the scattering structures resolved by our Capon algorithm.

\subsubsection{Capon and Fourier inversions}

Figure 5a shows the range-time-intensity (RTI) map of the echoes observed on 24 November 2005. The RTI map shows bottom-type irregularities starting around 19:15 LT at approximately $250 \mathrm{~km}$ altitude. Topside irregularities start to be observed around 20:45 LT, with echoes reaching as high as $650 \mathrm{~km}$ altitude.

The São Luís data consisted of cross-spectral measurements for four spectral bins, which allowed us to infer information about the line-of-sight Doppler velocity of the scatter- ers, in addition to their location. Two-dimensional in-beam radar images are constructed by stacking one-dimensional brightness distributions $B(\omega, \psi)$ estimated for each range gate (e.g., Hysell and Woodman, 1997).

Figure $5 \mathrm{~b}$ and $\mathrm{c}$ show the in-beam images produced by our algorithms for measurements made at 21:14:31 LT. The left panel shows the image produced by the Fourier method, while the right panel shows the image produced by the Capon method.

The estimated images are scaled by the SNR of the echoes. The Doppler information is encoded in the pixel colors. The green components represent small mean Doppler velocities. Red and blue components represent irregularities moving away and towards the radar, respectively.

Both images show a topside ESF structure that, as will be shown later, drifts from west (left-hand side) to east. The images also show that the structure is tilted to the west, and this is, presumably, caused by height variations in the zonal plasma drifts. More importantly, one can see that the Capon method produces a better-resolved image than the Fourier method. In some range gates, the Fourier images show artifacts in the brightness that are intrinsic to the method. For instance, the simulations in Fig. 3 show an increased brightness outside the scattering region produced by the sidelobes of the Fourier estimates even under high SNR conditions.

The Capon image shows the scattering region is narrower (in the zonal direction) than what is presented by the Fourier image. The Fourier image shows scattering features that are broad and diffuse; a result of the short baselines used for visibility functions. This is particularly true for the strong scattering channel seen above $350 \mathrm{~km}$ altitude. The colors also indicate that irregularities are ascending with large velocities within the scattering structure.

Finally, we must point out that the images were produced with the same number of incoherent integrations used in our simulations $(K=800)$. This requires an integration time of approximately $30 \mathrm{~s}$. The zonal motion of the irregularities during the integration time can affect the resolution of the images. Considering a zonal irregularity velocity of about $100 \mathrm{~m} \mathrm{~s}^{-1}$, the spatial resolution in the zonal direction will be limited to $3 \mathrm{~km}$, or approximately $0.5^{\circ}$ in the zenith angle for scatterers at $300 \mathrm{~km}$ altitude. Note, however, that the effects of irregularity zonal drifts in the angular resolution will be reduced at higher altitudes. Slower zonal drifts at lower altitudes (bottom-side $\mathrm{F}$ region) associated with the evening vortex (e.g., Kudeki and Bhattacharyya, 1999) will also reduce the degradation in the angular resolution.

\subsubsection{Zonal motion and multiple plumes}

Figure 6 shows a sequence of images created using the Capon method for measurements made between 20:50 and 21:03 LT. The sequence serves to illustrate the ability of the Capon method to produce images that provide accurate information about the dynamics of ESF irregularities. The im- 

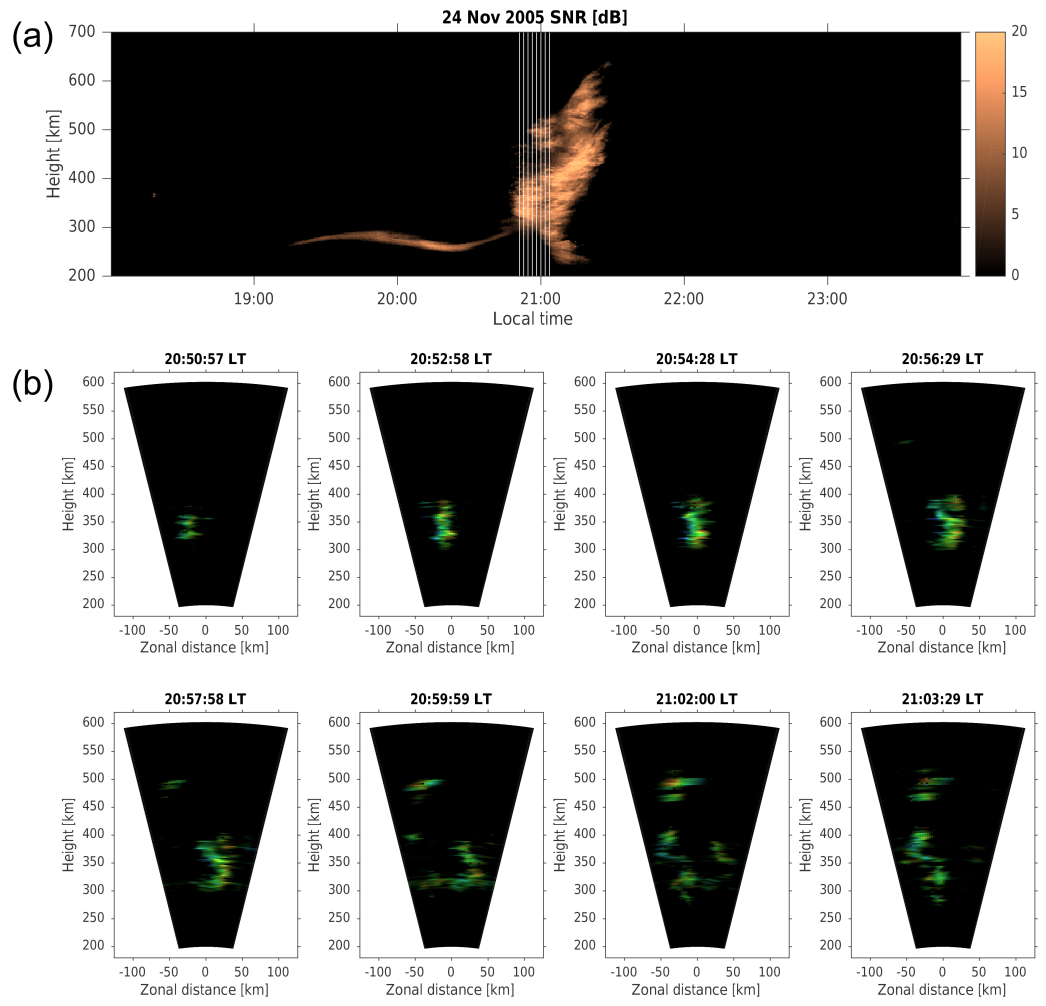

Figure 6. Panel (a) shows the RTI map of F region observations made by the São Luís radar on 24 November 2005 . Panel (b) shows a sequence of images obtained with the Capon method and interferometric observations. The time for each image is indicated above each panel and as vertical white lines in the RTI map.

ages show, initially, a scattering structure entering the field of view of the radar moving from the west. The sequence of images shows that the scattering structure moves in the zonal direction at a rate of about $130 \mathrm{~m} \mathrm{~s}^{-1}$. The scattering structure is thought to be embedded within (or along the walls of) large-scale plasma depletions. Therefore, its motion reflects the motion of the underlying large-scale depletion, which has been suggested to be a good tracer of the zonal plasma motion (Chapagain et al., 2013). We found that the direction and magnitude of the irregularity drift matches expectations based on previous observations. Measurements of the background plasma zonal drifts made by the Jicamarca incoherent scatter radar show average values around $120 \mathrm{~m} \mathrm{~s}^{-1}$ for similar times (21:00 LT) and solar flux conditions ( 90 SFU) (e.g., Fejer et al., 2005).

At 20:59:59 LT one can start to identify two vertically developed ESF structures, spaced in the zonal direction, within the radar field of view. The two structures are more clearly seen at 21:01:45 LT. The first (easternmost) structure reaches about $400 \mathrm{~km}$ altitude, while the second structure reaches over $500 \mathrm{~km}$ altitude. The measurements were made during low solar flux conditions when the altitudinal reach of ESF structures is limited (e.g., Gentile et al., 2006). Previous studies suggest that the zonal wavelength of the initial perturbation (seed wave) controls the spacing between plumes (e.g., Tsunoda and White, 1981; Makela et al., 2010; Huang et al., 2013). These studies focused, in general, on scale sizes of a few to several hundreds of kilometers. Hysell and Kudeki (2004), on the other hand, suggested that a collisional shear instability operating in the bottom-side equatorial $\mathrm{F}$ region can produce transient plasma perturbations with deca-kilometric zonal scale sizes. Our results show that ESF channels spaced by approximately $50 \mathrm{~km}$ (see Fig. 6 at 21:01:45 LT) can be detected, and these scale sizes should be taken into consideration in theories explaining the initiation and morphology of ESF structures.

We point our that the multiple structures seen in the inbeam images could not be inferred from the RTI map. The RTI map alone would suggest that a single radar plume passed over the radar site around 21:00 LT. Also, the detection of multiple plumes within the radar field of view is possible because of the large east-west beamwidths of the antennas used by the São Luís interferometer.

\subsubsection{Bifurcation and C-shaped structures}

Figure 7 shows another sequence of images obtained with the Capon algorithm but now for times between 21:03 and 21:13 LT. As shown in the previous sequence, the scattering region continues to move in the eastward direction. Around 

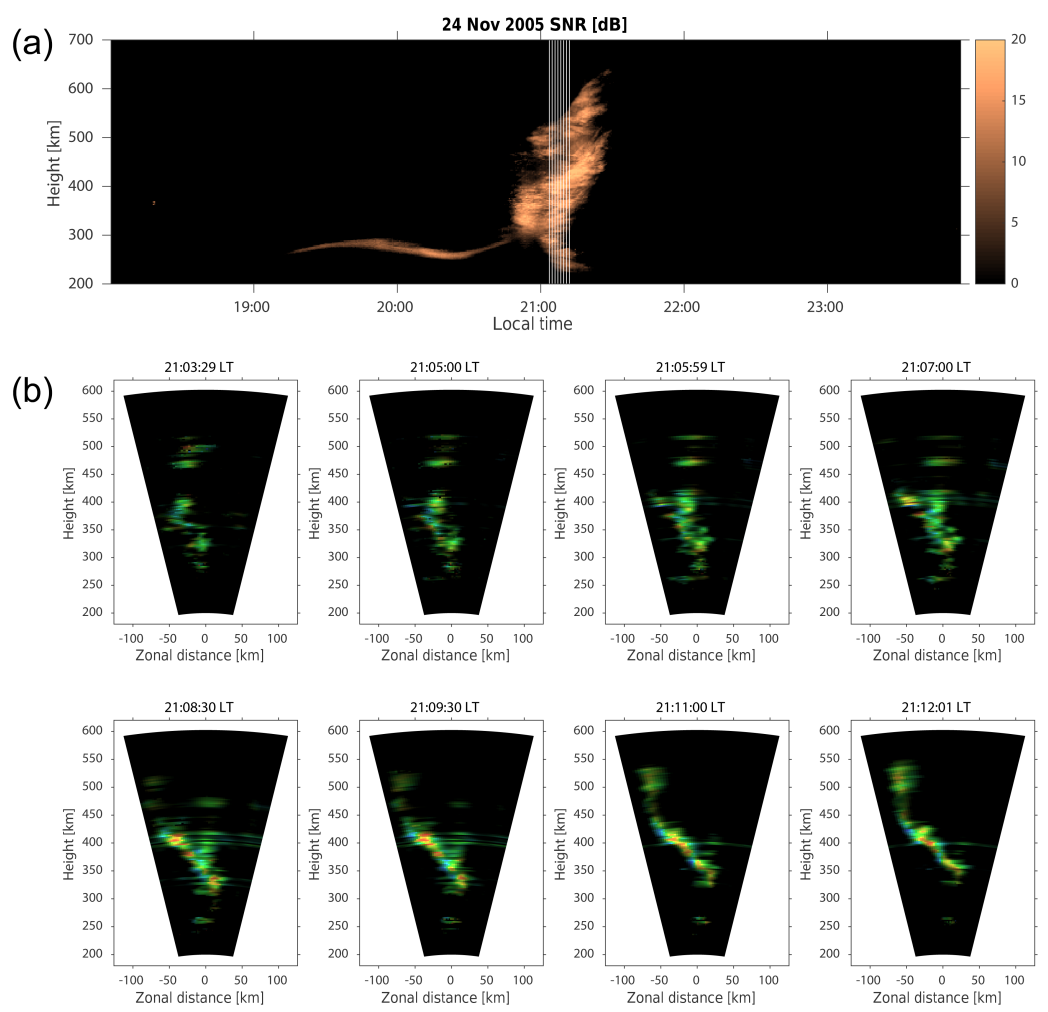

Figure 7. Panel (a) shows the RTI map of F region observations made by the São Luís radar on 24 November 2005 . Panel (b) shows a sequence of images obtained with the Capon method and interferometric observations. The time for each image is indicated above each panel and as vertical white lines in the RTI map.

21:07 LT, the imaging results allow us to identify a bifurcation of the scattering structure starting at about $375 \mathrm{~km}$ altitude. Bifurcation in ESF structures is thought to be caused by a secondary interchange instability occurring on the leading "head" of the ESF plasma depletion as it rises into the main F region and topside (e.g., Hysell, 2000; Yokoyama et al., 2015). One can see regions of large upward flows (red spots) within the first (east) branch of the bifurcation between 21:03 and 21:06 LT. Then, echoes are mostly broad and weaker. By 21:12 LT there are no echoes associated with the first branch. As the structure moves to the east, the images also show the development of the second (west) branch. Well-defined, large flows (red and blue spots) are seen again within the west channel as it reaches higher altitudes. By 21:12 LT, only the west branch is seen in the images.

In addition to bifurcation, the images produced by the Capon method are capable of resolving the $\mathrm{C}$-shape structure of the ionospheric perturbation, which has been predicted by numerical models of ESF (e.g., Zalesak et al., 1982) and observed using the ALTAIR steerable radar (e.g., Tsunoda, 1981). The sequence in Fig. 7 shows that the scattering structure is tilted to the east from about 200 to $300 \mathrm{~km}$ altitude. Then, above $300 \mathrm{~km}$, the tilt is westward. This is clearer in the image at 21:08:30 LT. The tilt is a signature of complexities in the height variation of the zonal plasma velocity. It suggests a maximum in the zonal velocity around $300 \mathrm{~km}$. This variability in the zonal drifts is caused by height variations in the vertical component of the electric field $\left(E_{L}\right)$, which in turn is controlled by variations, as a function of apex height, of flux-tube integration parameters such as Hall and Pedersen conductivities $\left(\Sigma_{\mathrm{H}}\right.$ and $\left.\Sigma_{\mathrm{P}}\right)$, conductivity-weighted zonal wind velocity $\left(U_{\phi}^{\mathrm{P}}\right)$, and equatorial zonal electric field $\left(E_{\phi}\right)$ and currents $\left(J_{L}\right)$ (Haerendel et al., 1992):

$E_{L}=\frac{\Sigma_{\mathrm{H}}}{\Sigma_{\mathrm{P}}} E_{\phi}-B U_{\phi}^{\mathrm{P}}+\frac{J_{L}}{\Sigma_{\mathrm{P}}}$,

where $B$ is the geomagnetic field intensity, which also varies with height.

\section{Conclusions}

We investigated the application of the Capon method (Capon, 1969; Palmer et al., 1998) to obtain in-beam images of scattering regions from measurements made by a small (max-

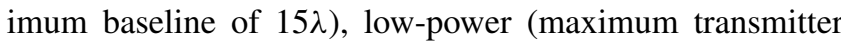
power of $8 \mathrm{~kW}$ ) coherent backscatter radar interferometer.

Numerical simulations were used to evaluate the performance of the Capon method. The numerical simulations show that, for broad scattering structures and the São Luís antenna configuration, the Capon method does not outperform 
the simple Fourier method. The simulations also show, however, that the strength of the Capon method lies in its ability to identify localized (narrow) scattering structures. We found that images with an angular resolution of a fraction of a degree can be obtained for typical equatorial spread F (ESF) measurement conditions found with the São Luís radar setup using the Capon algorithm. In practice, the resolution is also controlled by the magnitude of zonal irregularity drift during the integration time.

Following the simulation analyses, we applied the Capon method to actual measurements made by the São Luís interferometer during a typical ESF event detected on 24 November 2005. The Capon technique produces sharper images than those created by the Fourier method and better resolves the actual widths of the scattering structures.

Sequences of images show consistency from one inversion to the next indicating the robustness of the method. The sequence of images also shows the occurrence of ionospheric phenomena with better spatial and temporal resolution than would be possible with other types of instruments (e.g., scanning radars or airglow imagers). The images show scattering channels with zonal widths of a few kilometers. As expected from previous observations, the scattering structures move to the east as they evolve in time. Scattering channels spaced by only a few tens of kilometers in the zonal direction can be resolved, and their occurrence should be taken into consideration in theories and the description of ESF. The Capon images are also able to reveal the occurrence of bifurcation in ESF structures as well as variations in the zonal tilt of the scattering channels.

It is believed that the MaxEnt technique can produce images with more accuracy than the Capon method, particularly for conditions of low SNR (Yu et al., 2000). The Capon method, however, is computationally inexpensive with the potential to be used in near real-time observations, needed, for instance, during scientific rocket campaigns. Future work includes the implementation of compressed sensing (e.g., Harding and Milla, 2013) and MaxEnt algorithms for comparison with Capon inversions given the São Luís interferometer setup.

Data availability. The interferometric radar data used in this study are available upon request from Fabiano Rodrigues (email: fabiano@utdallas.edu).

Competing interests. The authors declare that they have no conflict of interest.
Acknowledgements. This work was supported by NSF (AGS1261107) and AFOSR (FA9550-13-1-0095). Eurico R. de Paula acknowledges the support from $\mathrm{CNPq}$ under process number 310802/2015-6. The authors would like to thank A. Cunha for their efforts in the operation and maintenance of the São Luís radar.

The topical editor, P. J. Erickson, thanks two anonymous referees for help in evaluating this paper.

\section{References}

Capon, J.: High resolution frequency-wavenumber spectrum analysis, Proc. IEEE, 57, 1408-1418, 1969.

Chapagain, N. P., Fisher, D. J., Meriwether, J. W., Chau, J. L., and Makela, J. J.: Comparison of zonal neutral winds with equatorial plasma bubble and plasma drift velocities, J. Geophys. Res.Space, 118, 1802-1812, doi:10.1002/jgra.50238, 2013.

Chau, J. L. and Woodman, R. F.: Three-dimensional coherent radar imaging at Jicamarca: comparison of different inversion techniques, J. Atmos. Sol.-Terr. Phy., 63, 253-261, doi:10.1016/S1364-6826(00)00142-5, 2001.

de Paula, E. R. and Hysell, D. L.: The São Luís $30 \mathrm{MHz}$ coherent scatter ionospheric radar: System description and initial results, Radio Sci., 39, RS1014, doi:10.1029/2003RS002914, 2004.

Farley, D. T.: Incoherent Scatter Correlation Function Measurements, Radio Sci., 4, 935-953, doi:10.1029/RS004i010p00935, 1969.

Farley, D. T. and Hysell, D. L.: Radar measurements of very small aspect angles in the equatorial ionosphere, J. Geophys. Res., 101, 5177-5184, doi:10.1029/95JA02640, 1996.

Fejer, B. G., de Souza, J., Santos, A. S., and Costa Pereira, A. E.: Climatology of $\mathrm{F}$ region zonal plasma drifts over Jicamarca, J. Geophys. Res., 110, A12310, doi:10.1029/2005JA011324, 2005.

Gentile, L. C., Burke, W. J., and Rich, F. J.: A climatology of equatorial plasma bubbles from DMSP 1989-2004, Radio Sci., 41, RS5S21, doi:10.1029/2005RS003340, 2006.

Haerendel, G., Eccles, J. V., and Cakir, S.: Theory for modeling the equatorial evening ionosphere and the origin of the shear in the horizontal plasma flow, J. Geophys. Res., 97, 1209-1223, 1992.

Harding, B. J. and Milla, M.: Radar imaging with compressed sensing, Radio Sci., 48, 582-588, doi:10.1002/rds.20063, 2013.

Huang, C.-S., de La Beaujardiére, O., Roddy, P. A., Hunton, D. E., Ballenthin, J. O., Hairston, M. R., and Pfaff, R. F.: Largescale quasiperiodic plasma bubbles: C/NOFS observations and causal mechanism, J. Geophys. Res.-Space, 118, 3602-3612, doi:10.1002/jgra.50338, 2013.

Hysell, D. L.: Radar imaging of equatorial F region irregularities with maximum entropy interferometry, Radio Sci., 31, 15671578, doi:10.1029/96RS02334, 1996.

Hysell, D. L.: An overview and synthesis of plasma irregularities in equatorial spread F, J. Atmos. Sol.-Terr. Phy., 62, 1037-1056, 2000.

Hysell, D. L. and Chau, J. L.: Optimal aperture synthesis radar imaging, Radio Sci., 41, RS2003, doi:10.1029/2005RS003383, 2006.

Hysell, D. L. and Kudeki, E.: Collisional shear instability in the equatorial F region ionosphere, J. Geophys. Res., 109, A11301, doi:10.1029/2004JA010636, 2004. 
Hysell, D. L. and Woodman, R. F.: Imaging coherent backscatter radar observations of topside equatorial spread F, Radio Sci., 32, 2309-2320, doi:10.1029/97RS01802, 1997.

Hysell, D. L., Milla, M. A., Condori, L., and Meriwether, J. W.: Data-driven numerical simulations of equatorial spread $\mathrm{F}$ in the Peruvian sector: 2. Autumnal equinox, J. Geophys. Res.-Space, 119, 6981-6993, doi:10.1002/2014JA020345, 2014.

Kintner, P. M., Ledvina, B. M., and de Paula, E. R.: GPS and ionospheric scintillations, Space Weather, 5, S09003, doi:10.1029/2006SW000260, 2007.

Kudeki, E. and Bhattacharyya, S.: Postsunset vortex in equatorial Fregion plasma drifts and implications for bottomside spread-F, J. Geophys. Res., 104, 28163-28170, doi:10.1029/1998JA900111, 1999.

Kudeki, E. and Franke, S. J.: Radar interferometer estimates of zonal drift variability during spread F, Geophy. Res. Lett., 13, 1117-1120, 1986.

Kudeki, E. and Sürücü, F.: Radar interferometric imaging of fieldaligned plasma irregularities in the equatorial electrojet, Geophy. Res. Lett., 18, 41-44, 1991.

Makela, J. J., Vadas, S. L., Muryanto, R., Duly, T., and Crowley, G.: Periodic spacing between consecutive equatorial plasma bubbles, Geophys. Res. Lett., 37, L14103, doi:10.1029/2010GL043968, 2010.

Palmer, R. D., Gopalam, S., Yu, T.-Y., and Fukao, S.: Coherent radar imaging using Capon's method, Radio Sci., 33, 1585-1598, doi:10.1029/98RS02200, 1998.

Rodrigues, F. S., Hysell, D. L., and de Paula, E. R.: Coherent backscatter radar imaging in Brazil: large-scale waves in the bottomside F-region at the onset of equatorial spread F, Ann. Geophys., 26, 3355-3364, doi:10.5194/angeo-26-3355-2008, 2008.

Rodrigues, F. S., Moraes, A. O., and dePaula, E. R.: Imaging equatorial spread $\mathrm{F}$ irregularities with the São Luis coherent backscatter radar interferometer, Radio Sci., 47, RSOL03, doi:10.1029/2011RS004929, 2012.
Shume, E. B., Rodrigues, F. S., Mannucci, A. J., and de Paula, E. R.: Modulation of equatorial electrojet irregularities by atmospheric gravity waves, J. Geophys. Res.-Space, 119, 366-374, doi:10.1002/2013JA019300, 2014.

Tsunoda, R. T.: Time evolution and dynamics of equatorial backscatter plumes 1 . Growth phase, J. Geophys. Res., 86, 139149, doi:10.1029/JA086iA01p00139, 1981.

Tsunoda, R. T. and White, B. R.: On the generation and growth of equatorial backscatter plumes: 1 . Wave structure in the bottomside F layer, J. Geophys. Res., 86, 3610-3616, 1981.

Woodman, R. F.: Coherent radar imaging: Signal processing and statistical properties, Radio Sci., 32, 2373-2391, doi:10.1029/97RS02017, 1997.

Woodman, R. F.: Spread F - an old equatorial aeronomy problem finally resolved?, Ann. Geophys., 27, 1915-1934, doi:10.5194/angeo-27-1915-2009, 2009.

Yokoyama, T., Shinagawa, H., and Jin, H.: Nonlinear growth, bifurcation and pinching of equatorial plasma bubble simulated by three-dimensional high-resolution bubble model, J. Geophys. Res.-Space, 119, 10474-10482, doi:10.1002/2014JA020708, 2015.

Yu, T.-Y., Palmer, R. D., and Hysell, D. L.: A simulation study of coherent radar imaging, Radio Sci., 35, 1129-1141, doi:10.1029/1999RS002236, 2000.

Zalesak, S. T., Ossakow, S. L., and Chaturvedi, P. K.: Nonlinear equatorial spread F: The effect of neutral winds and background Pedersen conductivity, J. Geophys. Res., 87, 151-166, doi:10.1029/JA087iA01p00151, 1982. 